\title{
On-Line Prediction of NBTI-induced Aging Rates
}

\author{
Baranowski, Rafal; Firouzi, Farshad; Kiamehr, Saman; Liu, Chang; \\ Tahoori, Mehdi; Wunderlich, Hans-Joachim
}

\author{
Proceedings of the ACM/IEEE Conference on Design, Automation Test in Europe \\ (DATE'15) Grenoble, France, 9-13 March 2015
}

url: http://dl.acm.org/citation.cfm?id=2755886

\begin{abstract}
Nanoscale technologies are increasingly susceptible to aging processes such as Negative-Bias Temperature Instability (NBTI) which undermine the reliability of VLSI systems. Existing monitoring techniques can detect the violation of safety margins and hence make the prediction of an imminent failure possible. However, since such techniques can only detect measurable degradation effects which appear after a relatively long period of system operation, they are not well suited to early aging prediction and proactive aging alleviation. This work presents a novel method for the monitoring of NBTI-induced degradation rate in digital circuits. It enables the timely adoption of proper mitigation techniques that reduce the impact of aging. The developed method employs machine learning techniques to find a small set of so called Representative Critical Gates (RCG), the workload of which is correlated with the degradation of the entire circuit. The workload of RCGs is observed in hardware using so called workload monitors. The output of the workload monitors is evaluated on-line to predict system degradation experienced within a configurable (short) period of time, e.g. a fraction of a second. Experimental results show that the developed monitors predict the degradation rate with an average error of only $1.6 \%$ at $4.2 \%$ area overhead.
\end{abstract}

\section{Preprint}

\section{General Copyright Notice}

This article may be used for research, teaching and private study purposes. Any substantial or systematic reproduction, re-distribution, re-selling, loan or sub-licensing, systematic supply or distribution in any form to anyone is expressly forbidden.

This is the author's "personal copy" of the final, accepted version of the paper published by ACM. ${ }^{1}$

\footnotetext{
${ }^{1}$ ACM COPYRIGHT NOTICE

(C)2015 ACM. Permission to make digital or hard copies of part or all of this work for personal or classroom use is granted without fee provided that copies are not made or distributed for profit or commercial advantage and that copies bear this notice and the full citation on the first page. Copyrights for components of this work owned by others than ACM must be honored. Abstracting with credit is permitted. To copy otherwise, to republish, to post on servers or to redistribute to lists, requires prior specific permission and/or a fee.
} 


\section{On-Line Prediction of NBTI-induced Aging Rates}

Rafal Baranowski*, Farshad Firouzi ${ }^{\dagger}$, Saman Kiamehr ${ }^{\dagger}$, Chang Liu*, Mehdi Tahoori ${ }^{\dagger}$, and Hans-Joachim Wunderlich*

* Institute of Computer Architecture and Computer Engineering, University of Stuttgart, Germany

$\dagger$ Karlsruhe Institute of Technology, Karlsruhe, Germany

Abstract-Nanoscale technologies are increasingly susceptible to aging processes such as Negative-Bias Temperature Instability (NBTI) which undermine the reliability of VLSI systems. Existing monitoring techniques can detect the violation of safety margins and hence make the prediction of an imminent failure possible. However, since such techniques can only detect measurable degradation effects which appear after a relatively long period of system operation, they are not well suited to early aging prediction and proactive aging alleviation.

This work presents a novel method for the monitoring of NBTI-induced degradation rate in digital circuits. It enables the timely adoption of proper mitigation techniques that reduce the impact of aging. The developed method employs machine learning techniques to find a small set of so called Representative Critical Gates (RCG), the workload of which is correlated with the degradation of the entire circuit. The workload of RCGs is observed in hardware using so called workload monitors. The output of the workload monitors is evaluated on-line to predict system degradation experienced within a configurable (short) period of time, e.g. a fraction of a second. Experimental results show that the developed monitors predict the degradation rate with an average error of only $1.6 \%$ at $4.2 \%$ area overhead.

Keywords-Representative critical gates, workload monitoring, aging prediction, NBTI

\section{INTRODUCTION}

As the scaling of technology nodes proceeds, Negative Bias Temperature Instability (NBTI) becomes a major threat to the reliability of VLSI devices [1]. NBTI consists in the oxide degradation of PMOS transistors that results in a gradual shift in the threshold voltage, which in turn causes an increased propagation delay. Eventually, NBTI stress may significantly increase the critical path delay and lead to timing violations.

Traditional approaches for NBTI monitoring measure degradation effects, i.e. provide an aggregated measure of the degradation that took place over a long period of time. They can be used to guide reactive techniques that manage the degradation effects, e.g. by frequency and voltage scaling [2] or adaptive body biasing [3]. Such monitoring techniques, however, are unable to track the degradation rate, i.e. the amount of stress caused by the currently running application. Degradation rate monitoring is crucial for the timely adoption of preventive techniques that alleviate aging, such as proactive frequency and voltage scaling [4] or dynamic cooling [5].

The goal of this work is to enable the monitoring of NBTI-induced degradation rate in digital circuits, i.e. to predict the increase of the critical path delay over a short period of time, long before any measurable degradation takes place. This kind of monitoring enables the adoption of novel proactive countermeasures that reduce the degradation rate, e.g. NBTI-aware scheduling, load balancing, frequency and voltage scaling, or guide the application of healing patterns [6].

In digital circuits, monitoring of the delay degradation rate is challenging for three reasons: (1) Direct aging rate estimation by consecutive measurements of the critical path delay is impractical over a short period of time as the delay increase is not measurable. (2) The degradation rate of a digital circuit depends on the degradation rate of each PMOS transistor on the current critical path, whereas the critical path may change over time. (3) The degradation rate of each PMOS transistor is a function of its state (or duty cycle) which in turn depends on the currently running application.

In this paper, we describe an innovative monitoring approach that combines workload monitoring with machine learning techniques. Our method is based on the monitoring of representative critical gates (RCG), the workload of which correlates with the degradation rate of the entire circuit. We provide an algorithm for finding a small set of RCGs, a method for the synthesis of RCG workload monitors, and an algorithm for online prediction of the delay degradation rate. Our experimental results show that the developed monitoring scheme predicts the degradation rate with an average error of only $1.6 \%$ at the expense of a modest $4.2 \%$ area overhead.

The rest of this paper is organized as follows: In the next section, we give the problem statement together with an overview of the developed monitoring scheme. Section III defines critical gates and provides the algorithm to select representative critical gates (RCGs). The synthesis of workload monitors for RCGs is described in Section IV. Section V deals with the construction of regression models that are used on-line to predict the degradation rate. The accuracy and overhead of the monitoring scheme is evaluated in Section VI.

\section{OVERVIEW}

\section{A. Problem Statement}

NBTI causes a gradual increase in gate delays and results in performance degradation of digital circuits. The aging rate at time $t$ is defined as $\delta D(t) / \delta t$, where $D(t)$ is the length of the critical path at time $t$. The NBTI-induced aging rate is a function of many technology parameters, temperature, and the duty cycle of transistors, i.e. the ratio between transistor stress time to the total operating time.

NBTI is a long term phenomenon which impacts the circuit delay after a long time. To characterize the currently running application and guide aging alleviation techniques, it is sufficient to average the degradation rate over a period of several milliseconds to several minutes. Therefore, the goal of our work is to approximate the average aging rate over a given period of time $T_{0}$. At time $t=k T_{0}, k \in \mathbb{N}^{+}$, the average aging rate is calculated as $\Delta D / T_{0}$, where $\Delta D$ is the increase (degradation) of the circuit delay within the time interval $\left[t-T_{0}, t\right)$. The parameter $T_{0}$, i.e. the length of the time window over which the aging rate is averaged, must be configurable to suit different applications of aging rate monitoring.

\section{B. Developed Monitoring Approach}

The degradation rate monitoring scheme is presented in Fig. 1. The monitored circuit is augmented with a workload 
monitor that observes a subset of the circuit's primary and pseudo-primary inputs, and a temperature sensor. Based on the state of the primary and pseudo-primary inputs, the workload monitor predicts the current stress of each representative critical gate (RCG) of the monitored circuit. Note that the RCGs are not monitored directly to limit the impact of monitoring on circuit performance. The outputs of the workload monitor are aggregated over a short period of time and used to predict the current degradation rate, i.e., the increase in the circuit delay over the recent period. A software component is responsible for the evaluation of the degradation rate based on the aggregated output from the workload monitor and the temperature sensor.

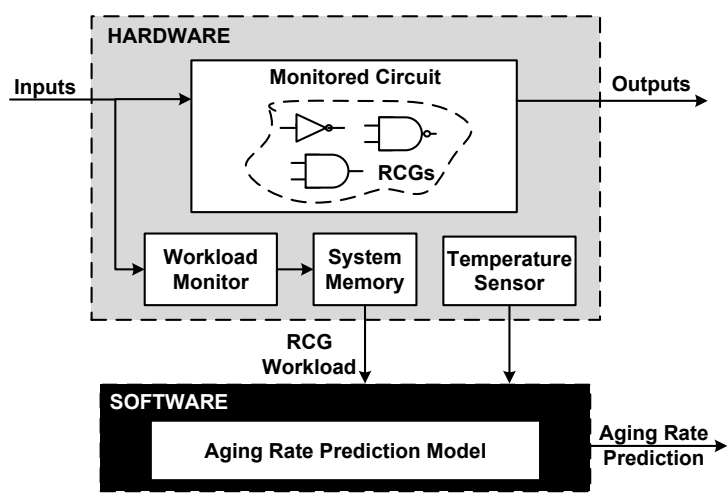

Fig. 1. Principle of the degradation rate monitoring

The monitoring method comprises design-time and on-line algorithms and activities, as shown in Fig. 2. At design-time, we characterize the target standard cell library w.r.t. NBTI aging and subject the circuit to an NBTI-aware Static Timing Analysis (STA). Based on STA results, we identify the Critical Gates (CG), i.e. gates that belong to critical paths or paths that may become critical due to aging. From the set of CGs, we select a small set of representative critical gates (RCGs), the delay degradation of which is correlated with the degradation of the entire circuit. Next, we synthesize workload monitors that predict the NBTI-relevant stress of RCGs. Finally, a regressionbased aging rate prediction model is constructed.

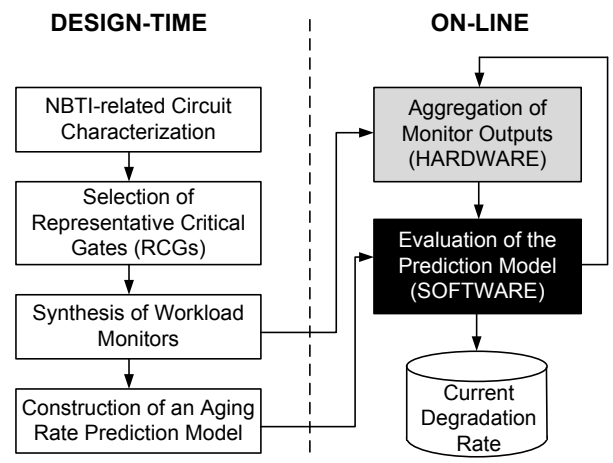

Fig. 2. Design-time algorithms and on-line activities for aging rate prediction

In a running system, the workload monitors continuously observe the stress experienced by the RCGs. Together with the current temperature, this information is periodically fed to the aging rate prediction model. The aging rate model is evaluated in software, ideally during the idle time of any available processing unit.

\section{REPRESENTATIVE CRITICAL GATES (RCG)}

\section{A. Critical Gate Selection}

We define the slack of a gate as the difference between the delay of the longest path through that gate and the critical path delay of the circuit. If the slack of a gate does not exceed a given threshold, the gate is called critical gate (CG). The set of CGs is found using an aging-aware timing analysis based on [7]. The threshold can be adjusted based on the delay degradation rate of the circuit.

We define the workload of a critical gate as the duty cycles of its constituent PMOS transistors. Circuit degradation rate is predicted by monitoring the workload of selected CGs, as discussed below.

\section{B. RCG Selection Algorithm}

Since monitoring of all the CGs in a circuit is infeasible, it is crucial to find a small set of representative critical gates (RCGs). The workload of RCGs must be highly correlated with the NBTI-induced delay degradation of the circuit. RCG selection problem can be formulated as follows: for a given set of CGs $V$, identify a minimal subset $F \subseteq V$ such that the circuit delay degradation rate can be predicted with sufficient accuracy only from the workload of gates in $F$ and the chip temperature. Solving this problem requires all possible circuit applications and aging histories be considered, which is infeasible. Instead, we select the set of RCGs by evaluating the correlation between the delays of RCGs and the circuit delay.

The set of RCGs is found using the wrapper method for feature selection [8]. The training set consists of sets of degraded CG delays and the corresponding degraded circuit delay obtained for random applications. The following merit function is used to score each subset $S \subseteq V$ consisting of $k$ features: $k \overline{r_{c f}} / \sqrt{k+k(k-1) \overline{r_{f f}}}$, where $\overline{r_{f f}}$ is the correlation between features, and $\overline{r_{c f}}$ is the average correlation between features and circuit delay. The correlations $\overline{r_{f f}}$ and $\overline{r_{c f}}$ are calculated as minimum description length [8]: $E(A)+E(B \mid A)$, where $\mathrm{E}$ is entropy while $\mathrm{A}$ and $\mathrm{B}$ are either feature or delay degradation.

\section{WORKLOAD MONITORING}

A workload monitor is a combinational circuit that observes a subset of the circuit's primary inputs and pseudo-primary inputs (register outputs) and generates one output per PMOS transistor of each RCG. An output of the monitor is " 1 " when the corresponding transistor experiences NBTI stress $\left(\mathrm{U}_{\mathrm{GS}}<0\right)$, and it is " 0 " otherwise. Since the monitor observes only primary and pseudo-primary inputs, it has minimal impact on the timing of the monitored circuit.

The problem of monitor synthesis is defined as follows: Given a circuit with $n$ primary inputs and $m$ pseudo-primary inputs, a set of RCGs, and target monitoring accuracy $A_{T}$ expressed in percent, construct a workload monitor with minimal area overhead such that each monitor output provides the correct response for at least $A_{T} \cdot 2^{n+m}$ input patterns.

In principle, workload monitors can be synthesized using the technique presented in [9]. Our experimental results show, however, that this approach results in prohibitive area overhead 
that often exceeds $100 \%$ for the usual number of RCGs required for accurate aging rate prediction. In the following, we describe a novel heuristic method for the synthesis of workload monitors with affordable area overhead: Initially, we construct an exact monitor that provides correct response for all input patterns. Next, we build approximate monitors with reduced size by iterative reduction of the exact monitor.

The exact monitor is simply a copy of the monitored circuit in which (1) all gates that do not belong to the transitive fan-in cone of any RCG are removed, (2) additional gates are added to generate outputs that are " 1 " whenever their corresponding PMOS transistors experience NBTI stress $\left(\mathrm{U}_{\mathrm{GS}}<0\right)$.

An approximate monitor is constructed by iterative removal of gates from the exact monitor at the cost of reduced accuracy. A gate is removed if its output controllability and observability is low enough to guarantee the target monitoring accuracy $A_{T}$. The removal of gates is modeled by the injection of stuck-at faults in the monitor. For each injected fault, we evaluate the resulting accuracy, as well as the area reduction due to fault injection and constant propagation. In every iteration, we select a fault that does not violate the target monitoring accuracy $A_{T}$ and results in the lowest size of the monitor.

Formally, the approximate monitors are synthesized as follows: Let $M$ be the exact monitor with an output set $\left\{o_{1}, o_{2}, o_{3}, \ldots, o_{G}\right\}$. Iterate:

1) Generate a structurally collapsed stuck-at fault set $\left\{f_{1}, f_{2}, f_{3}, \ldots, f_{K}\right\}$ for $M$.

2) For each fault $f_{i}$, create a structural copy of $M$, denoted $M_{i}$, with injected fault $f_{i}$.

3) Using Monte Carlo simulation, evaluate the accuracy of each monitor output $o_{j}$ of $M_{i}$, denoted $a_{i}\left(o_{j}\right)$, i.e. the ratio of input patterns for which the output $o_{j}$ in $M_{i}$ matches the corresponding output in the exact monitor.

4) For each faulty monitor $M_{i}$, calculate $A_{i}=\operatorname{MIN}_{j=1}^{G} a_{i}\left(o_{j}\right)$.

5) Terminate if $\operatorname{MAX}_{i=1}^{K} A_{i}<A_{T}$.

6) For each $M_{i}$ with $A_{i} \geq A_{T}$, perform fault/constant propagation, remove the gates with constant outputs, and simplify the gates with constant inputs.

7) Approximate the size of each $M_{i}$, denoted $S_{i}$, as the total area of standard cells in $M_{i}$.

8) Choose monitor $M_{t}, 1 \leq t \leq K$, such that $A_{t} \geq A_{T}$ and $S_{t}=\operatorname{MIN}_{i=1}^{K} S_{i}$; assign $M:=M_{t}$, repeat from step 1 .

\section{PREdiction of the Degradation Rate}

\section{A. Off-Line Construction of the Aging Rate Model}

For on-line prediction, it is necessary to model the relationship between the output of workload monitors and the delay degradation rate of the circuit. The aging rate model is a linear regression of the following form: $\Delta D=W \cdot \beta+\epsilon$, where $\Delta D$ is the increase in circuit delay, $W$ is a vector of average RCG workloads from the monitor, while the vector $\beta$ and the scalar $\epsilon$ are regression coefficients. To fit the model, we use the ordinary least squares (OLS) technique.

\section{B. On-Line Model Evaluation}

To evaluate the aging rate prediction model, the duty cycles of PMOS transistors in RCGs must be averaged over the time period $T_{0}$. To this end, each output of the workload monitor is aggregated in hardware. In the simplest case, one counter per monitor output is used to count up the number of clock cycles in which the output is " 1 ", i.e. cycles in which the corresponding PMOS transistor experiences NBTI stress.

To reduce the monitoring cost, an existing memory system is reused and systematic sampling methods are applied: Just a single counter with multiplexed input is used regardless of how many RCGs are monitored. The counter is attached to each monitor output for the time period of $T_{0} / G$, where $G$ is the total number of monitor outputs. The minimal counter length is hence $L:=\left\lceil\log _{2} \frac{T_{0}}{G \cdot T_{c l k}}\right\rceil$, where $T_{c l k}$ is the minimal period of the system clock. The content of the counter is sent to the main system memory with a period of $T_{0} / G$ using Direct Memory Access (DMA). The memory demand is $G \cdot L$ bits. For instance, assuming 30 monitor outputs, an evaluation period of $1 \mathrm{~s}$, and $1 \mathrm{GHz}$ clock, only a single 25-bit counter and 94 bytes of memory are required.

Since the aging rate prediction is executed relatively seldom, e.g. with a period of several hundred milliseconds to minutes, it is performed in software during the idle time of any currently available processing unit. The processing unit reads the average chip temperature as well as the aggregated output of the workload monitors from the system memory. These data are then used to evaluate the aging rate prediction model. The only requirement on the processing unit is that it supports efficient addition and multiplication of fixed or floating point numbers.

\section{Vi. Evaluation}

\section{A. Experimental Setup}

We evaluate the on-line prediction technique on a set of ISCAS' 89 benchmark circuits. We define an application of a benchmark circuit as a set of primary input signal probabilities. The Nangate $45 \mathrm{~nm}$ open cell library [10] is used to synthesize the benchmark circuits and monitors. We assume that the benchmarks are part of a system with a temperature sensor, a memory system with DMA and free capacity of several KB, and a processing unit that can be reused in the idle time to evaluate the prediction model. We exploit the reaction-diffusion (R-D) NBTI model proposed in [11]. The worst case NBTIinduced delay degradation is assumed to be $10 \%$ over 3 years for a simple inverter and the parameters of the NBTI model are set accordingly. Due to limited space, we provide results for a constant system temperature.

\section{B. Impact of Application on Aging Rate}

To study the effect of different applications on the aging rate, 5000 random sets of primary input signal probabilities are considered. Fig. 3 shows the range by which the degradation rate differs across the applications for each benchmark circuit. The range is calculated as: $[\max (\Delta D)-\min (\Delta D)] / \min (\Delta D)$, where $\max (\Delta D)$ and $\min (\Delta D)$ are respectively the maximum and minimum NBTI-induced delay degradation over all applications. Since the effect of the application on the amount of NBTI-induced delay degradation can be as large as $40 \%$, it is crucial to monitor the circuit's workload for an accurate aging rate prediction. 


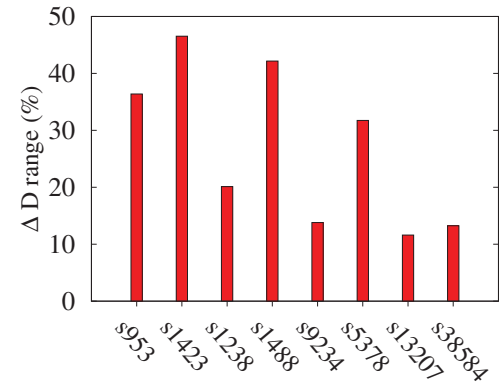

Fig. 3. Normalized range of NBTI-induced delay degradation across different applications

\section{Validation Experiments}

To evaluate the accuracy of the developed method, 4500 of the application sets are used as a training set for linear regression, and 500 sets are used for validation. For each application, the accurate NBTI-induced delay degradation $\Delta D_{\text {calc }}$ is computed based on the NBTI-aware timing analysis [7]. The corresponding on-line estimation $\Delta D_{\text {est }}$ is obtained by feeding the average outputs of the workload monitor to the aging rate prediction model. The prediction accuracy is calculated as normalized root-mean-square error (NRMSE):

$$
N R M S E=\frac{\sqrt{\sum_{i=1}^{n}\left(\Delta D_{\text {est }_{i}}-\Delta D_{\text {calc }_{i}}\right)^{2}}}{\sqrt{n}\left(\Delta D_{\text {calc }_{\text {max }}}-\Delta D_{\text {calc }_{\text {min }}}\right)},
$$

where $n$ is the number of applications (in this case 500).

\section{Accuracy of Aging Prediction}

Table I shows the number of CGs for all benchmark circuits assuming a CG slack threshold of 5\%. Simulation results show that $5 \%$ slack threshold gives a good trade-off between the number of CGs and prediction accuracy. As described in Section III-B, machine-learning is used to find a set of RCGs to decrease the monitoring overhead. The column 4 of Table I shows the number of RCGs, which is less than the number of CGs by a factor of four on average.

We evaluate the prediction accuracy with two sets of workload monitors: the first one can monitor the workload with $100 \%$ accuracy and the other with $80 \%$ accuracy (see Section IV). As shown in Fig. 4, the average NRMSE of the proposed technique is around $1.8 \%$ and $3.6 \%$ for $100 \%$ and $80 \%$ monitors, respectively. These results correspond to a mean error of $0.8 \%$ and $1.6 \%$ for $100 \%$ and $80 \%$ monitors, respectively. While the inaccuracy of $80 \%$ monitors is higher, their area overhead is less as discussed below.

\section{E. Hardware Overhead}

The area overhead of the workload monitors is presented in Table I: the size of the benchmark circuits is given in the fifth column, while the two following columns show the monitoring

TABLE I: AREA OVERHEAD OF THE DEVELOPED MONITORING SCHEME
\begin{tabular}{|l|r|r|r|r|r|r|}
\hline Circuit & \#Gates & \#CGs & \#RCGs & $\begin{array}{c}\text { Area } \\
{\left[\mu m^{2}\right]}\end{array}$ & \multicolumn{2}{|c|}{ Monitoring overhead } \\
\cline { 5 - 7 } & & & $A_{T}=100 \%$ & $A_{T}=80 \%$ \\
\hline s953 & 683 & 26 & 18 & 479.86 & $24.39 \%$ & $8.31 \%$ \\
s1423 & 824 & 116 & 44 & 808.64 & $22.13 \%$ & $10.23 \%$ \\
s1238 & 881 & 47 & 22 & 546.10 & $56.06 \%$ & $7.54 \%$ \\
s1488 & 902 & 27 & 14 & 571.37 & $13.73 \%$ & $3.49 \%$ \\
s9234 & 1725 & 92 & 15 & 2388.68 & $15.93 \%$ & $1.61 \%$ \\
s5378 & 2926 & 50 & 16 & 1934.88 & $6.61 \%$ & $2.36 \%$ \\
s13207 & 4074 & 162 & 14 & 5159.87 & $15.10 \%$ & $0.20 \%$ \\
s38584 & 18142 & 59 & 21 & 16861.74 & $0.48 \%$ & $0.30 \%$ \\
\hline
\end{tabular}

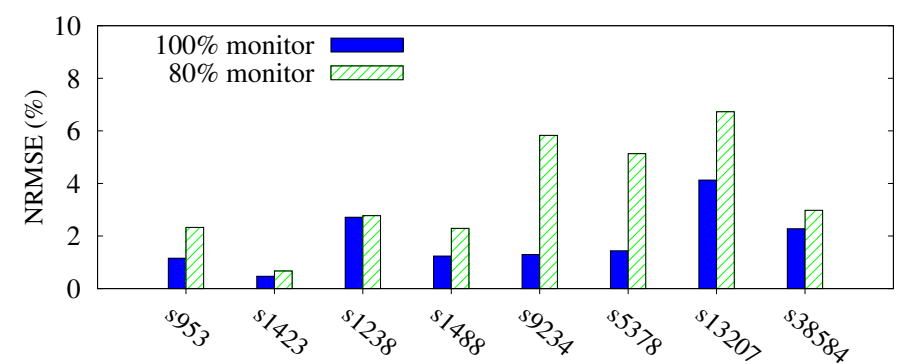

Fig. 4. Degradation rate prediction accuracy. NRMSE: normalized root-meansquare error

overhead for monitors with the target accuracy of $100 \%$ and $80 \%$ (cf. Section IV). The area overhead of the $80 \%$ monitors, which were shown to provide sufficient accuracy, is below $2.4 \%$ for circuits with more than 1000 gates. The monitoring overhead scales well with the circuit size: for the two largest benchmarks, the overhead is below $0.3 \%$.

\section{F. Runtime}

As mentioned in Section $\mathrm{V}$, the prediction technique consists of the off-line monitor synthesis and construction of the aging rate model, and the on-line model evaluation. The runtime of the off-line part is only few hours for the largest circuit. The online prediction time is negligible as it involves simple software vector multiplication that is executed relatively seldom.

\section{CONCLUSION}

Since the NBTI effect in advanced technology nodes strongly depends on the system application and workload, on-line prediction of the degradation rate is crucial to enable effective aging alleviation. We present a novel method for aging rate prediction which is based on workload monitoring and machine learning techniques. The monitoring technique enables online prediction of the degradation rate caused by the currently running application. Experimental results show that this method delivers sufficient accuracy at an affordable area overhead which decreases with the size of the monitored circuit.

\section{ACKNOWLEDGMENT}

This work was supported by the German Research Foundation (DFG) under grants TA 782/9-1 and WU 245/13-1 (RMBIST).

\section{REFERENCES}

[1] M. Alam et al., "A Comprehensive Model of PMOS NBTI Degradation," Microelectronics Reliability, vol. 45, no. 1, pp. 71-81, 2005.

[2] M. Nakai et al., "Dynamic Voltage and Frequency Management for a Low-Power Embedded Microprocessor," IEEE Journal of Solid-State Circuits, vol. 40, no. 1, pp. 28-35, 2005.

[3] S. Kumar et al., "Adaptive Techniques for Overcoming Performance Degradation due to Aging in Digital Circuits," in Proc. IEEE Asia and South Pacific Design Automation Conf. (ASP-DAC), 2009, pp. 284-289.

[4] C. Zhuo et al., "Process Variation and Temperature-Aware Reliability Management," in Proc. Design, Automation and Test in Europe (DATE), 2010, pp. 580-585.

[5] E. Mintarno et al., "Optimized Self-Tuning for Circuit Aging," in Proc. Design, Automation and Test in Europe (DATE), 2010, pp. 586-591

[6] F. Firouzi et al., "NBTI Mitigation by Optimized NOP Assignment and Insertion," in Proc. Design, Automation Test in Europe (DATE), March 2012, pp. 218-223.

[7] F. Firouzi et al., "Incorporating the Impacts of Workload-Dependent Runtime Variations into Timing Analysis," in Proc. Design, Automation and Test in Europe (DATE), 2013, pp. 1022-1025.

[8] M. A. Hall, "Correlation-based feature selection for machine learning," Ph.D dissertation, The University of Waikato, 1999.

[9] R. Baranowski et al., "Synthesis of Workload Monitors for On-Line Stress Prediction," in Proc. IEEE Intl. Symp. on Defect and Fault Tolerance in VLSI and Nanotechnology Systems (DFT), 2013, pp. 137-142.

[10] Nangate 45nm Open Cell Library v1.3, http://www.nangate.com

[11] S. Bhardwaj et al., "Predictive Modeling of the NBTI Effect for Reliable Design," in Proc. IEEE Custom Integrated Circuits Conf. (CICC), 2006, pp. 189-192. 